\title{
Simulation Toolbox for Studying Energy Consumption in Wired Networks
}

\author{
Anne-Cécile Orgerie*, Betsegaw Lemma Amersho ${ }^{\dagger}$, Timothée Haudebourg ${ }^{\ddagger}$, Martin Quinson ${ }^{\ddagger}$, \\ Myriana Rifai ${ }^{\S}$, Dino Lopez Pacheco ${ }^{\S}$ and Laurent Lefèvre \\ * CNRS, IRISA, Rennes, France - Email: anne-cecile.orgerie@irisa.fr \\ † Université Rennes 1, France \& Aalto University, Finland - Email: betsegaw.amersho@irisa.fr \\ $\ddagger$ ENS Rennes, IRISA, Rennes, France - Email: \{timothee.haudebourg, martin.quinson\}@ens-rennes.fr \\ $\S$ Université Nice Sophia Antipolis, I3S, France - Email: \{mrifai, dino.lopez\}@ unice.fr \\ ฯ Inria, LIP, ENS Lyon, University of Lyon, France - Email: laurent.lefevre@inria.fr
}

\begin{abstract}
Networking infrastructures are considered to consume as much energy as terminal end-user equipment or datacenters. While energy consumption of wireless networks is a matter of concern since their beginning, it is not the case for wired networks as they do not rely on batteries, but on plugged equipment. Yet, facing growing consumption, energyefficient techniques start to be implemented in wired networks. However, measuring the end-to-end energy consumption of wired networking infrastructures remains a real challenge for network operators and scientists. This article presents the ECOFEN (Energy Consumption mOdel For End-to-end Networks) framework which allows to support precise simulation of energy consumption of large-scale complex wired networks. The experimental validation shows that Ecofen provides accurate energy consumption values.
\end{abstract}

Index Terms-Energy consumption, simulation, wired networks, green device, ns3

\section{INTRODUCTION}

Networking infrastructures are considered to consume as much energy as terminal end-user equipment or datacenters [3]. But contrary to the two last families of consumers, networks can be very complex by nature with remote or legacy devices. Thus measuring end-to-end energy consumption of networking infrastructures can be unreachable or at least remains a real challenge for network operators and scientists. Simulation of energy consumption of networks is an alternative way in order to understand the energy impact of networking scenarios. This simulation approach has been widely studied in wireless open-source context where relevant devices are battery based and need to optimize their energy consumption [8], [11]. But such simulation frameworks dedicated for energy usage of wired infrastructures are mainly designed by network operators for specific networks. Based on ns-2 simulator, the Ecofen framework (End-to-end energy Cost mOdel and simulator For Evaluating power consumption in large-scale Networks) has been proposed in 2011 [7]. In 2013, Ecofen, has been refined and re-designed for ns3 [5] simulator, a discrete-event simulator for Internet systems, targeted primarily for research and educational use. Since then, Ecofen has been used by several research teams for studying various networking infrastructures. Ecofen has become a mature framework able to simulate complex networking scenarios in order to study energy consumption and energy leverages in networks.

The main goal of the Ecofen toolbox is to provide a simulating environment for large-scale wired networks where users can obtain the energy consumption of their new protocols, algorithms and frameworks involving different types of technologies and equipment. It is endowed with several energy models, and several representative network devices are pre-defined using energy consumption values found in the literature.

The article is organized as follows. Section II presents the related works. Section III introduces the Ecofen model and simulator. Section IV analyzes a validation of the framework. Section V concludes and presents future work.

\section{RELATED WORK AND CONTEXT}

Several network simulators are available for studying and analyzing wired networks like OMNET++ discrete event simulator [6] or the ns-* frameworks. One of the most famous network simulator is the ns-2 simulator [4]. Ns-2 is a discreteevent network simulator and has been widely employed to study several transport and network layer protocols over wiredand wireless-based networks. While the high popularity of ns2 catalyzed the implementation of several protocol models, the simplicity of the implemented models might lead to results different to the ones that would be obtained in real networks [2], [10]. This is the case for instance of the by-default TCP models in ns-2 and this is why a Linux-based code, known as the TCP-Linux ns-2 agent, has been implemented for instance.

To tackle the accuracy problems in the ns- 2 results, the ns- 3 network simulator has been developed [5]. Note that the ns-2 and the ns-3 simulators are incompatible. Ns-3 is a discreteevent network simulator, built as a $\mathrm{C}++$ library which may be statically or dynamically linked to a $\mathrm{C}++$ main program that defines the simulation topology and starts the simulator. In ns-3, the accuracy of the results are improved with the introduction of several Linux protocols implementations and kernel modules in its framework (e.g. the TCP/IP Linux implementation). Additionally, external Linux-based user-land tools can be plugged to the ns- 3 simulator by means of the 
ns-3-dce API. Hence, iperf or still Quagga can be used in ns-3 simulations.

\section{THE ECOFEN FRAMEWORK}

This section describes the Ecofen features and implementation. The Ecofen simulator has an architecture comprising three layers: energy models, green functions and network management. The energy models define the energy consumption values for each device and thus the relation between energy consumption and traffic load. The green functions are energyaware levers to enable devices to be put to sleep or to be completely turned off for example. They come with their own energy model (energy required to switch off an interface for instance).

The state-of-the-art services provide network-wide functions such as energy-aware routing protocols or network management algorithms. Such services are not yet implemented in Ecofen but can be added by users using the green functions (to switch off nodes for instance, or to get the energy consumption of each device).

The simulator provides energy consumption values at different levels: for the entire studied network (to compare networkwide frameworks); for a given equipment (to compare nodelevel optimizations); for a given transfer or flow (to evaluate the cost of a service or a data transfer). It is implemented within the ns-3 network simulator, and can thus benefit from all the protocols already implemented in this simulator.

\section{A. Energy models}

To model the energy consumption at a low level, the Ecofen simulator provides different energy models and different energy profiles for several categories of devices. The consumption of each equipment is divided between the chassis consumption and the consumption of the interfaces (or ports).

Different models are proposed for the interfaces and for the moment, only one model is proposed for the chassis. These models can be configured for each modeled device using measured values or values taken from literature. So, according to the values used for the ports and for the chassis, users can simulate Ethernet cards, routers, switches, etc. Currently in Ecofen, the ns-3 supported models include Point ToPointNetDevice and CsmaNetDevice for the net device models (interfaces), and Ipv4 and Ipv6 for the IP layer.

Currently, only one model is implemented for the nodes (chassis consumption). It allows users to specify an OnConso: the node's power consumption (in Watts) when it is on, and an OffConso: the node's power consumption when the node is switched off. These values can be put to 0 if required. Each node of the simulation has its own model (so nodes can use different power values within the same simulation).

Currently, three energy models are available for the net devices (per interface consumption):

- basic, that allows user to specify the OnConso (OffConso): the interface's power consumption when it is on (respectively off).
- linear, that allows user to specify the OffConso (IdleConso): the interface's power consumption when it is off (respectively on but idle, i.e. no traffic), and a ByteEnergy value: the energy consumed per processed Byte (incoming or outgoing).

- complete, that allows user to specify OffConso (IdleConso): the interface's power consumption when it is off (respectively on but idle); RecvByteEnergy (SentByteEnergy): the energy consumed per Byte received (respectively sent); and RecvPktEnergy: the energy consumed per packet received (respectively sent).

The last model relies on the fact that the energy consumption of some interfaces depends on the traffic in two ways: it depends on the number of Bytes (because they need to be written in memory for instance), and it depends on the number of packets (because they need to be processed: reading the headers for instance) [9]. Table I summarizes the possible attributes per energy model, with the unit and the data format of each attribute.

TABLE I

ATTRIBUTES FOR EACH ENERGY MODEL

\begin{tabular}{|c|c|c|c|}
\hline Model name & Attribute name & Expected units & Format \\
\hline \multicolumn{4}{|c|}{ Node energy models } \\
\hline \multirow[t]{2}{*}{ basic } & OnConso & Watts & double \\
\hline & OffConso & Watts & double \\
\hline \multicolumn{4}{|c|}{ Net device energy models } \\
\hline \multirow[t]{2}{*}{ basic } & OnConso & Watts & double \\
\hline & OffConso & Watts & double \\
\hline \multirow[t]{3}{*}{ linear } & IdleConso & Watts & double \\
\hline & OffConso & Watts & double \\
\hline & ByteEnergy & nanoJoules per Byte & double \\
\hline \multirow[t]{6}{*}{ complete } & IdleConso & Watts & double \\
\hline & OffConso & Watts & double \\
\hline & RecvByteEnergy & nanoJoules per Byte & double \\
\hline & SentByteEnergy & nanoJoules per Byte & double \\
\hline & RecvPktEnergy & nanoJoules per packet & double \\
\hline & SentPktEnergy & nanoJoules per packet & double \\
\hline
\end{tabular}

\section{B. Green functions}

The Ecofen simulator provides basic tools (or green functions) to design complex energy-efficient frameworks, protocols and algorithms that act at the network level. These basic tools are energy-efficient techniques that can be applied at the node level, currently on/off techniques, ALR and LPI [1]. The user is responsible to implement the coordination framework on top of these functions. For example, the simulator provides a function to switch off the devices, but the user has to determine when this function is used (e.g. autonomic decision or coordinated plan).

Ecofen enables to switch off ports. This functionality can be used to switch off unused ports or to simulate failures. Switching on and off require time and energy. In the Ecofen simulator, these two parameters can be modified by users. Ecofen provides several functions to the users: NetDeviceSwitchoff (NetDeviceSwitchon) to switch off (respectively on) an interface at a given date; 
LinkSwitchoff (LinkSwitchoff) to switch off (respectively on) a link at a given date.

Prior to use these functions, the user should precise an energy model to employ to switch on and off the interfaces. This model allows users to specify the energy parameters that will be used to switch on and off interfaces: SwitchoffEnergy (SwitchonEnergy): the total amount of energy needed to switch off (respectively on) the interface and SwitchoffDuration (SwitchonDuration): the time needed to switch off (respectively on) the interface. A switch off interface cannot receive nor send traffic.

Along with interface switching functions, Ecofen provides similar tools to switch on and off entire nodes: NodeSwitchoff (NodeSwitchon) to switch off (respectively on) a node at a given date. Similarly to the interface model, for switching a node, the user should first define an energy model. When a node is switched off, all its interfaces are unreachable.

\section{VALIDATION}

Both the linear and complete models can produce values of power consumption as a function of traffic. The basic model, on the other hand, produces power consumption values based on the ON or OFF state of a node, it does not consider network traffic. Therefore we describe the validation experiments for both the linear and the complete models in this section. The basic procedure for the validation experiment is first to simulate, using Ecofen, power consumption in response to traffic sent or received and then to compare the results against data obtained from literature where actual measurements are conducted.

\section{A. Validating the Linear Model}

Sivaraman et al. [9] provide the results of power consumption experiments on real equipment. These experiments show the linear relationship that exists between traffic volume (in Mbps) and power consumption (in Watts) for fixed packet sizes of 100, 576, 1000, and 1500 Bytes. Furthermore, the linear fit equations for each of the packet size are also provided.

The authors' intention in these experiments is to determine values of the per-byte and the per-packet processing energy consumption, however, our intention is to use the per-byte energy consumption value that they have experimentally determined and to use it in Ecofen to get power consumption values for a given volume of traffic. We then compare the results we obtained with the linear fit models provided in [9]. In their experiments, the authors used three kinds of hardware devices: (1) NetFPGA router card that has four 1 Gbps Ethernet ports, (2) IXIA hardware traffic-generator for generating packets with the desired packet-size and data-rate, and (3) highfidelity oscilloscope for measuring the power consumed by the NetFPGA card as a consequence of the packets send or received.

In this validation experiment we wish to simulate the experiments conducted by Sivaraman et al. as closely as possible.

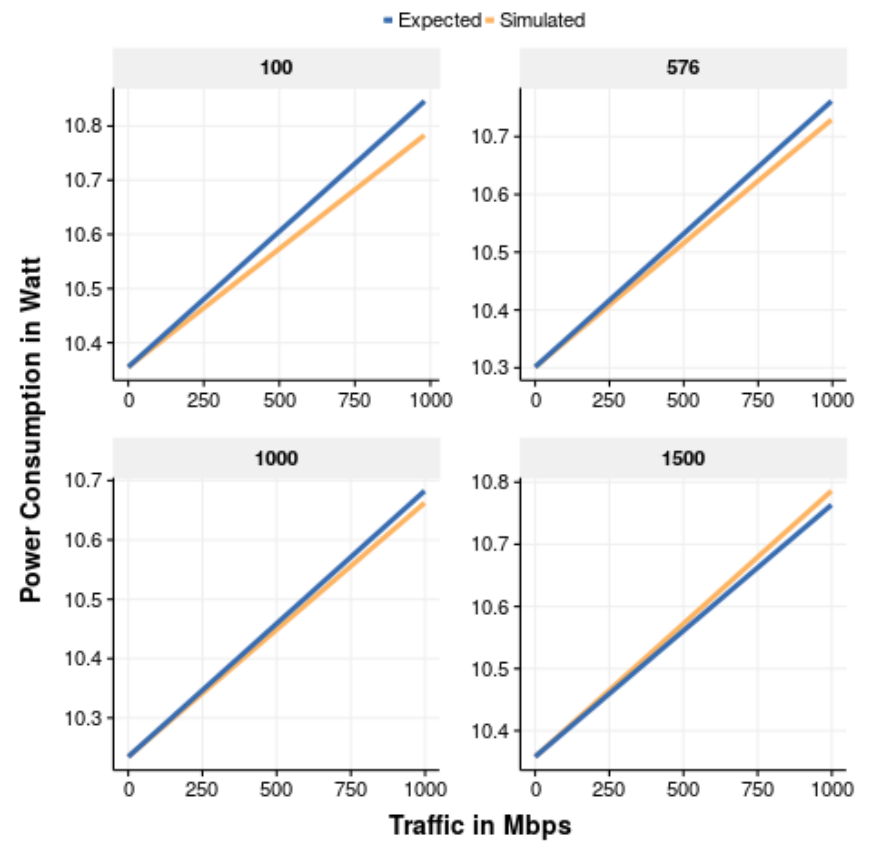

Fig. 1. Power consumption vs data-rate comparison between expected (or measured) values (in blue) and simulated values (in yellow) for a fixed packet size of 100, 576, 1000, and 1500 Bytes

With this in mind, we setup, in our ns-3 simulation script, a three node simple network with first and third nodes connected to the second node. All the three nodes are connected to each other by links that have maximum bandwidth capacity of 1 Gbps and delay of $10 \mathrm{~ms}$.

For the generated traffic volume in the simulation, we used uniform random number generator provided by ns-3. Finally, each experiment was run 40 times, each time with a different value for the random number generator. The results obtained are depicted in Figure 1. In the graph the expected power consumption values from the linear fit models shown in [9] along with the simulated values for each of the packet sizes (100, 576, 1000, and 1500 bytes) are displayed.

Visually, the simulated and the expected values seem to agree very well, even though a gap draws between them as the data rate increases (specially true for the case of 100bytes packet lengths). Hence, to better assess the accuracy of our results, we run unpaired t-test statistical test using the produced data. The summary of this test is shown in Table II.

The 95\% confidence interval values shown in Table II of difference in mean between the measured and simulated values are very close to zero and in fact zero is also one of the values. According to the P-values, the null hypothesis that the difference in mean between the simulated and the expected values is zero is not rejected.

The conclusion in this validation test is that the linear model of Ecofen is accurate in predicting the power consumed by NetFPGA router for a given volume of traffic. 
TABLE II

UNPAIRED T-TEST RESULTS FOR SIMULATED AND MEASURED POWER CONSUMPTION VALUES FOR ECOFEN'S LINEAR

\begin{tabular}{|c|c|c|c|c|}
\hline $\begin{array}{c}\text { Packet } \\
\text { Size }\end{array}$ & $\begin{array}{c}\text { Confidence } \\
\text { Interval of } \\
\text { difference in } \\
\text { mean }\end{array}$ & $\begin{array}{c}\text { Mean of } \\
\text { Expected }\end{array}$ & $\begin{array}{c}\text { Mean of } \\
\text { Simulated }\end{array}$ & P-Value \\
\hline 100 & {$[-0.027,0.110]$} & 10.640 & 10.599 & 0.230 \\
\hline 576 & {$[-0.039,0.082]$} & 10.544 & 10.523 & 0.480 \\
\hline 1000 & {$[-0.043,0.073]$} & 10.466 & 10.451 & 0.6131 \\
\hline 1500 & {$[-0.062,0.048]$} & 10.566 & 10.573 & 0.796 \\
\hline
\end{tabular}

\section{B. Validating the Complete Model}

Roughly, in this validation experiment, we have used the same experimental configuration and procedure as that of the linear validation experiment that we have described in the previous section.

One of the main difference between the complete and the linear model of Ecofen is that the complete model distinguishes between the received and sent bytes. Which means that different energy consumption values can be assigned to bytes based on the direction of transfer. The linear model, on the other hand, assigns same value for both. The other main difference is that the complete model considers the packet processing energy consumption cost both for the sent and received packets.

Sivaraman et al. [9] also conducted experiments to determine energy consumption values for per-byte receive or transmit and per-packet processing. The experimentally determined values for per-byte receive is $1.3 \mathrm{~nJ}$, for per-byte transmit is $2.1 \mathrm{~nJ}$, and for per-packet processing is $197.2 \mathrm{~nJ}$.

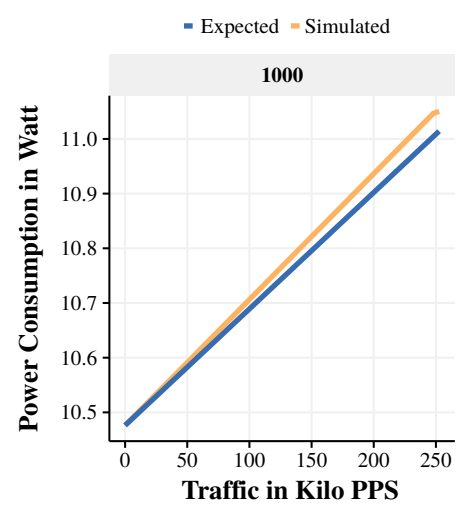

Fig. 2. Power consumption vs data-rate comparison between expected (or measured) values (in blue) and simulated values (in yellow) for a fixed packet size of 1000 Bytes for the sending end

The results for the sending and receiving are shown in Figures 2 and 3, respectively.

Table III shows the unpaired t-test result for one packetsizes in the transmitting side (Tx) and for two packet-sizes in the receiving side $(\mathrm{Rx})$.

Again in this case, the $95 \%$ confidence interval values shown in Table III of difference in mean between the measured and simulated values are very close to zero and in fact zero

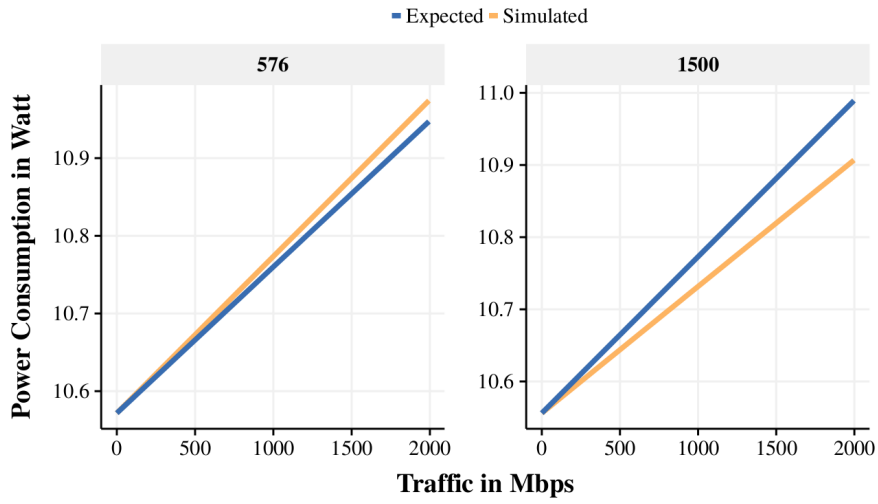

Fig. 3. Power consumption vs data-rate comparison between expected (or measured) values (in blue) and simulated values (in yellow) for a fixed packet size of 576 and 1500 Bytes for the receiving end

TABLE III

UNPAIRED T-TEST RESULTS FOR SIMULATED AND MEASURED POWER CONSUMPTION VALUES FOR ECOFEN'S COMPLETE MODEL

\begin{tabular}{|c|c|c|c|c|c|}
\hline $\begin{array}{c}\text { Packet } \\
\text { Size }\end{array}$ & End & $\begin{array}{c}\text { Confidence } \\
\text { interval of } \\
\text { difference in } \\
\text { mean }\end{array}$ & $\begin{array}{c}\text { Mean } \\
\text { of Ex- } \\
\text { pected }\end{array}$ & $\begin{array}{c}\text { Mean } \\
\text { of Sim- } \\
\text { ulated }\end{array}$ & P-Value \\
\hline 576 & $\mathrm{Rx}$ & {$[-0.067,0.039]$} & 10.770 & 10.784 & 0.598 \\
\hline 1500 & $\mathrm{Rx}$ & {$[-0.010,0.095]$} & 10.778 & 10.736 & 0.114 \\
\hline 1000 & $\mathrm{Tx}$ & {$[-0.096,0.053]$} & 10.750 & 10.773 & 0.560 \\
\hline
\end{tabular}

is also one of the values. According to the P-values, the null hypothesis that the difference in mean between the measured and the simulated values is zero is not rejected.

The conclusion from this validation experiment is also the same as the previous one, the Ecofen's complete energy consumption model accurately predicts power consumed by NetFPGA router for a given volume of sent or received traffic.

\section{CONClusion}

Studying and understanding the energy consumption of the network infrastructures can be very complex due to the presence of multiple forwarding devices and middle-boxes, potentially spread over multiple countries. Simulation tools are good candidates to study the energy consumption of networks. However, while multiple solutions exist to study the energy consumption in wireless networks (e.g. in 802.11 networks) through simulation, little attention has been put over wired networks.

The Ecofen model, proposed in this article, aims at filling the void of energy modeling in wired networks. Ecofen was initially designed to be integrated into the ns- 2 simulator. Currently, we have redesigned and updated Ecofen to be integrated within the ns-3 network simulator. Since ns-3 is able to run native Linux code to provide results close to the real life, ns-3 with Ecofen is expected to provide valuable insights about the energy consumption of real networks and energy efficient protocols. 


\section{REFERENCES}

[1] T. Haudebourg and A.-C. Orgerie, "On the Energy Efficiency of Sleeping and Rate Adaptation for Network Devices," in International Conference on Algorithms and Architectures for Parallel Processing (ICA3PP), 2017.

[2] G. F. Lucio, M. Paredes-farrera, E. Jammeh, M. Fleury, and M. J. Reed, "Opnet modeler and ns-2: Comparing the accuracy of network simulators for packet-level analysis using a network testbed," in In 3rd WEAS International Conference on Simulation, Modelling and Optimization (ICOSMO), 2003, pp. 700-707.

[3] Nokia, "Global what-if analyzer of network energy consumption," http: //gwatt.net, 2016.

[4] ns-2. [Online]. Available: https://www.isi.edu/nsnam/ns/

[5] ns3 network simulator. [Online]. Available: http://www.nsnam.org/ ns-3-11/

[6] OMNeT++ Discrete Event Simulator. [Online]. Available: https: //omnetpp.org/

[7] A.-C. Orgerie, L. Lefèvre, I. Guérin-Lassous, and D. Lopez Pacheco, "Ecofen: an end-to-end energy cost model and simulator for evaluating power consumption in large-scale networks," in SustaInet: First International Workshop on Sustainable Internet and Internet for Sustainability (in conjunction with WoWMoM), 2011.

[8] P. Serrano, A. Garcia-Saavedra, G. Bianchi, A. Banchs, and A. Azcorra, "Per-Frame Energy Consumption in 802.11 Devices and Its Implication on Modeling and Design," IEEE/ACM Transactions on Networking, vol. 23, no. 4, pp. 1243-1256, 2015.

[9] V. Sivaraman, A. Vishwanath, Z. Zhao, and C. Russell, "Profiling per-packet and per-byte energy consumption in the NetFPGA Gigabit router," in 2011 Conference on Computer Communications Workshops (INFOCOM Workshops), 2011, pp. 331-336.

[10] D. X. Wei and P. Cao, "Ns-2 tcp-linux: An ns-2 tcp implementation with congestion control algorithms from linux," in Proceeding from the 2006 Workshop on Ns-2: The IP Network Simulator, ser. WNS2 '06. New York, NY, USA: ACM, 2006. [Online]. Available: http://doi.acm.org/10.1145/1190455.1190463

[11] H. Wu, S. Nabar, and R. Poovendran, "An Energy Framework for the Network Simulator 3 (NS-3)," in International ICST Conference on Simulation Tools and Techniques (SIMUTools), 2011, pp. 222-230. 